\title{
Modeling geophysical complexity: a case for geometric determinism
}

\author{
C. E. Puente ${ }^{1}$ and B. Sivakumar ${ }^{2}$ \\ ${ }^{1}$ Department of Land, Air \& Water Resources, University of California, Davis, USA \\ ${ }^{2}$ Griffith School of Engineering, Griffith University, Nathan, QLD 4111, Australia
}

Received: 3 February 2006 - Published in Hydrol. Earth Syst. Sci. Discuss.: 10 July 2006

Revised: 21 November 2006 - Accepted: 20 December 2006 - Published: 17 January 2007

\begin{abstract}
It has been customary in the last few decades to employ stochastic models to represent complex data sets encountered in geophysics, particularly in hydrology. This article reviews a deterministic geometric procedure to data modeling, one that represents whole data sets as derived distributions of simple multifractal measures via fractal functions. It is shown how such a procedure may lead to faithful holistic representations of existing geophysical data sets that, while complementing existing representations via stochastic methods, may also provide a compact language for geophysical complexity. The implications of these ideas, both scientific and philosophical, are stressed.
\end{abstract}

\section{Introduction}

The study of complex data sets is at the core of geophysical research. This has resulted in the use of a variety of techniques aimed at characterizing and modeling such sets. With the development of ideas, a variety of qualifiers (e.g. autocorrelation function, power spectrum, multifractal spectrum, probability distribution function, information function, chaotic invariant properties, etc.) have been used for such purposes, and the model's goodness has been defined in their ability to preserve such quantities.

As illustrated in Fig. 1, many data sets (e.g. rainfall and runoff time series in hydrology) exhibit high irregularity, non-trivial intermittency, long-term persistence, power-law power spectrum scaling and altogether "intrinsic randomness," which suggest, in a natural way, the usage of stochastic models, such as multiplicative cascades with random multipliers, to represent such "1/f ${ }^{\beta}$ noises" (e.g. Lovejoy and Schertzer, 1990; Veneziano et al., 2000). The ability of the stochastic models to reasonably represent important (statistical) characteristics of these data sets and the reasonably good

Correspondence to: C. E. Puente

(cepuente@ucdavis.edu) predictions reported on their underlying evolutions have further strengthened our view on the usefulness and appropriateness of such models.

This article argues that, given the irregularity present in geophysical data sets, such stochastic modeling may be supplemented with chaotic and other deterministic modeling to provide a more complete representation of the records and processes at hand. Besides the now classical use of phasespace reconstruction techniques (e.g. Packard et al., 1980; Takens, 1981) developed in nonlinear dynamics to qualify the intrinsic dimensionality of sets (e.g. Rodriguez-Iturbe et al., 1989; Tsonis et al., 1993; Sivakumar, 2000, 2004; Sivakumar et al., 2001), this article makes a case for a geometric representation of geophysical records as derived distributions of deterministic multifractal measures via simple deterministic fractal functions (Puente, 1992, 1994a), a novel deterministic procedure that may be useful in archiving data and in elucidating their dynamics.

\section{The fractal-multifractal procedure and geophysical applications}

This deterministic geometric procedure was inspired by the simply quantized layering of energy dissipation in fully developed turbulence (Meneveau and Sreenivasan, 1987) and by the automatic generation of the inherent multiplicative cascade while constructing fractal interpolating functions (Barnsley, 1988).

As illustrated in Fig. 2, the fractal-multifractal procedure transforms a turbulence-related measure over $x$, say $d x$ (i.e., a multifractal one found via a cascade and, hence, one without a density), into the unique measure $d y$, over $y$, defined as the derived distribution of $d x$ via a fractal function $f$ (Puente, 1992, 1994a). The ideas turned out to provide, in a way reminiscent of Plato's notions of "reality" as "shadows", a host of interesting patterns over $y$, whose peculiar shapes suggested, also in a natural manner, their usage as suitable geometric

Published by Copernicus GmbH on behalf of the European Geosciences Union. 

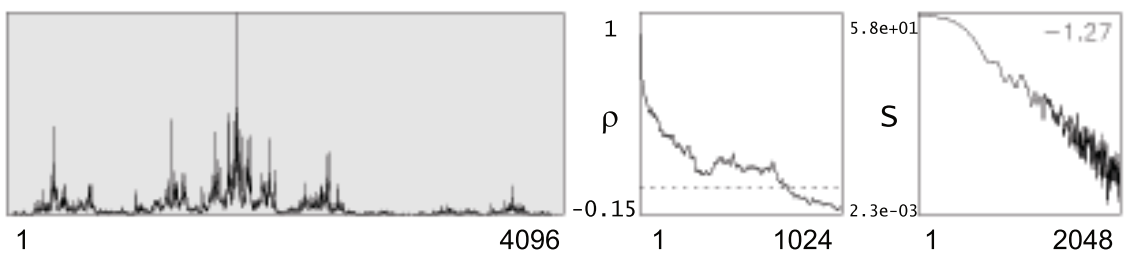

Fig. 1. A prototypical complex data set made of $2^{12}$ data points, followed by its autocorrelation function and its (log-log) power spectrum, with scaling exponent $\beta=1.27$.

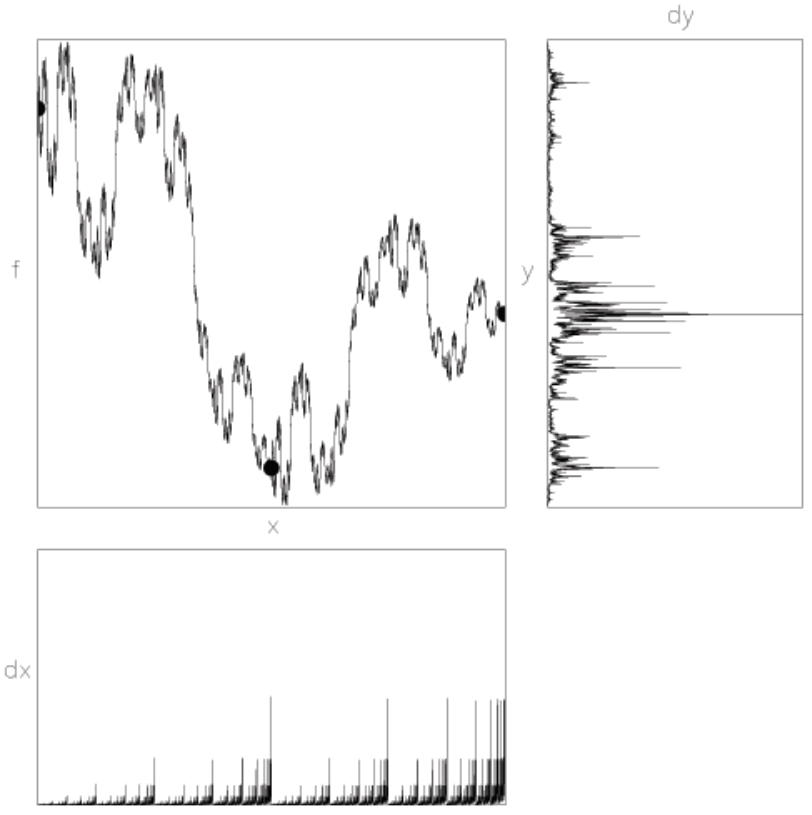

Fig. 2. An example of the fractal-multifractal procedure. The measure $d y$ is obtained adding the corresponding values of $d x$ for horizontal crossings of the mapping $f$.

models for geophysical data sets, including rainfall (Puente, 2004).

It so happens that the time series in Fig. 1 corresponds to $d y$ in Fig. 2. Specifically, such a complex set is found, at any resolution, as the unique invariant measure over $y$ (at a projection angle of zero degrees) generated by the two simple affine mappings, from the plane to the plane,

$w_{1}(x, y)=\left(\frac{1}{2} x,-0.51 x+d_{1} y\right)$

$w_{2}(x, y)=\left(\frac{1}{2} x+\frac{1}{2}, 0.03 x+d_{2} y-0.35\right)$

with the scaling parameters $d_{1}=-0.8$ and $d_{2}=-0.6$, when such mappings are iterated in an independent fashion according to a biased coin such that $w_{1}$ is used $30 \%$ of the time and $w_{2}$ the remaining $70 \%$. As illustrated in Fig. 2, these notions also define a fractal function that passes by the three points
$\{(0,0),(1 / 2,-0.35),(1,-0.2)\}$ whose graph has a fractal dimension of 1.485 , and the invariant (and hence deterministic) measure $d x$, as identified in turbulence studies (Puente and Obregón, 1999). As a way of clarification, it should be emphasized that although it may appear to a casual reader that the obtained patterns may depend on the "coin" mentioned above, such is not the case, since the successive iterations are just a suitable Monte Carlo approach that always converges to the same deterministic pattern (Barnsley, 1988).

By varying the parameters of the geometric construction, that is, the points by which a given fractal function passes (which could contain more than three points), the scaling parameters that determine the fractal dimension of the transformation, the iteration frequencies that define a parent (multinomial) multifractal over $x$, and the projection angle (other than zero degrees in the $y$ direction), one may indeed find a plethora of interesting patterns sharing the observed features of natural data sets (Puente, 2004). These resemble geophysical sets such as rainfall records and width functions of natural channels (usually modeled via stochastic cascades; e.g. Veneziano et al., 2000), among others (e.g. Puente and Obregón, 1996; Obregón et al., 2002; Puente and Sivakumar, 2003) and both chaotic and stochastic signals (with $1 / \mathrm{f}^{\beta}$ power spectra), as classified via phase-space reconstruction techniques (Puente et al., 2002).

\section{Scientific and philosophical implications}

As the advent of chaos theory has resulted in a debate of determinism vs. stochasticity as possible competing mechanisms to model natural data sets (e.g. Osborne and Provenzale, 1989; Koutsoyiannis and Pachakis, 1996; Sivakumar, 2000; Schertzer et al., 2002; Sivakumar et al., 2002a, b), it is pertinent to explain where the geometric ideas herein may fit within such a debate.

It ought to be stressed first that the fractal-multifractal procedure was not conceived as a means to endorse determinism and hence disprove randomness, but rather as a possible new framework for geophysical (hydrologic) complexity. The idea was developed as the first author attempted to employ extensions of fractal interpolating functions to represent mountain profiles from which to study the evolution of river networks (unsuccessfully) and when he later realized 
that the natural measure generated via the iterations over $\mathrm{x}$ correspond to the spiky measures found in atmospheric turbulence and generated via deterministic multiplicative cascades. Such realization triggered the question: if turbulence along one line is just a permutation of a rather simple multiplicative cascade, could it be that rainfall and other turbulence-driven processes are also simple enough to be transformations (fractional integrations) of such turbulence? As seen in Fig. 2, and as illustrated in the diverse applications already mentioned and in others, the ideas turned out to be sensible indeed in one and higher dimensions (Puente 1994b; Puente et al., 2001a, b), even if a complete physical explanation for the procedure remains a challenge.

It should also be noted that the Platonic approach has not been developed to add to the aforementioned determinism vs. stochasticity debate, although the label of determinism may suggest otherwise. That the outcomes of the fractal-multifractal approach are deterministic is unquestionable; but they may also be interpreted, again in a natural way, as specific realizations of existing stochastic processes, as soundly developed elsewhere (e.g. Lovejoy and Schertzer, 1990; Veneziano et al., 2000). In this context, the geometric approach provides an alternative way of generating interesting sets, which may be used as simulations to drive a variety of water resource applications.

Clearly, however, there are noticeable differences between such stochastic approaches and the geometric methodology explained herein. While the latter, as applied to one data set, naturally tries to find an underlying multiplicative (multinomial) process which when fractally transformed provides a representation of a whole normalized set, stochastic approaches, by construction, concentrate on relevant statistical properties (e.g., power spectrum, multifractal spectrum, codimension function) to find a suitable cascade mechanism that may generate them as a realization. While the classical approaches can not control the specific realization and instead concentrate on relevant statistics of the sets, the geometric procedure, once an inverse problem is solved, aims at the overall geometry of the sets, one that if properly captured includes also its statistics. This makes the geometric procedure all the more ambitious.

Although producing similar outcomes, there are two potential features of the geometric procedure (absent by definition from the more classical approaches) that we believe deserve further study as they may result in relevant methodological breakthroughs.

First, a Platonic methodology (similar to the one herein but defined possibly via alternative transformations not using affine mappings) furnishes us with the real possibility of encoding holistically complete data sets, with rather substantial compression ratios. This can be readily noticed in Fig. 2, as the rather complex set therein is totally described by only 9 parameters. For, even if it is argued that the geometric approach uses "many more" parameters than the stochastic representations based on faithful fittings of co-dimension func- tions, the latter, in its "characterization" of the record's intermittency structure, can not uniquely define a given realization, which the geometric approach can in principle do, including records that may be termed chaotic or stochastic (Puente et al., 2002), as mentioned earlier.

Second, a geometric approach may open new vistas to study the dynamics of geophysical processes. The evolution of records may perhaps be discerned in the compressed parameter space of subsequent sets, as has been found in a contamination problem when the geometric ideas are extended to produce complex patterns over two dimensions (Puente et al., 2001a, b). Notwithstanding the difficulties involved in thinking in a surrogate space as done in other disciplines (e.g. strings in theoretical physics), the notions may perhaps provide a dual representation for understanding dynamics, one that may prove useful as it has the potential of capturing the ever important details (i.e., timing and magnitude) present in the data sets at hand.

Whether simplicity can be found at the root of complexity remains a tremendous challenge in science (see, however, Wainright and Mulligan, 2004; for a similar issue in environmental modeling). Undoubtedly, geophysical complexity is very hard to quantify and as such there are, no doubt, opportunities for further improvement. In regards to the geometric ideas herein, more research is needed in trying to solve a complex inverse optimization problem and in finding ways by which physical knowledge, as defined via conservation principles and differential equations, may be coupled with the geometric ideas. It is our hope that such issues will have a successful ending.

\section{Conclusions}

A geometric procedure aimed at modeling complex geophysical data sets as derived distributions of multifractal measures via fractal functions has been reviewed. It has been argued, both scientifically and philosophically, that such an approach may provide a suitable language for geophysical complexity, one that may be useful to simulate distinct phenomena of diverse types and one that one day may lead to a new vision to study geophysical dynamics. We envision that this fractal-based approach and other procedures aiming to capture mathematical morphology (e.g. Maragos, 1989) would result in improved understandings of complex natural patterns, thresholds and their dynamics.

Acknowledgements. The work presented in this article was supported in part by the USEPA via Grant GAD \# R824780 and by NASA under grant NAG5-7441. Comments by Demetris Kouysoyiannis on an earlier version of this manuscript are greatly appreciated.

Edited by: M. Sivapalan 


\section{References}

Barnsley, M. F.: Fractals Everywhere, Academic Press, San Diego, USA, 1988.

Lovejoy, S. and Schertzer, D.: Multifractals, universality classes and satellite and radar measurements of cloud and rain fields, J. Geophys. Res. 95, 2021-2034, 1990.

Maragos, P.: Pattern spectrum and multiscale shape representation, IEEE Trans. Pattern Anal. Machine Intell., 11(7), 701-716, 1989.

Meneveau, C. and Sreenivasan, K. R.: Simple multifractal cascade model for fully developed turbulence, Phys. Rev. Lett., 59, 1424 1427, 1987.

Obregón, N., Sivakumar, B., and Puente, C. E.: A deterministic geometric representation of temporal rainfall: sensitivity analysis for a storm in Boston, J. Hydrol., 269(3-4), 224-235, 2002.

Osborne, A. R. and Provenzale, A.: Finite correlation dimension for stochastic systems with power-law spectra, Physica D, 35, 357-381, 1989.

Packard, N. H., Crutchfield, J. P., Farmer, J. D., and Shaw, R. S.: Geometry from a time series, Phys. Rev. Lett., 45(9), 712-716, 1980.

Puente, C. E.: Multinomial multifractals, fractal interpolators, and the Gaussian distribution, Phys. Lett. A, 161, 441-447, 1992.

Puente, C. E.: Deterministic fractal geometry and probability, Int. J. Bifurcations Chaos, 4(6), 1613-1629, 1994a.

Puente, C. E.: A fractal-multifractal approach to geostatistics. In Geostatistics for the Next Century, R. Dimitrakopoulos (Editor), Kluwer Academic Publishers, Dordrecht, 476-487, 1994b.

Puente, C. E.: A universe of projections: may Plato be right?, Chaos, Soliton. Fract., 19(2), 241-253, 2004.

Puente, C. E. and Obregón, N.: A deterministic geometric representation of temporal rainfall: Results for a storm in Boston, Water Resour. Res., 32(9), 2825-2839, 1996.

Puente, C. E. and N. Obregón: A geometric Platonic approach to multifractality and turbulence, Fractals, 7(4), 403-420, 1999.

Puente, C. E., Obregón., N., and Sivakumar, B.: Chaos and stochasticity in deterministically generated multifractal measures, Fractals, 10(1), 91-102, 2002.

Puente, C. E., Robayo, O., Diaz, M. C., and Sivakumar, B.: A fractal-multifractal approach to groundwater contamination. 1 . Modeling conservative tracers at the Borden site, Stoch. Env. Res. Risk Asses., 15(5), 357-371, 2001a.
Puente, C. E., Robayo, O., and Sivakumar, B.: A fractal-multifractal approach to groundwater contamination. 2. Predicting conservative tracers at the Borden site, Stoch. Env. Res. Risk Asses., 15(5), 372-383, 2001b.

Puente, C. E. and Sivakumar, B.: A deterministic width function model- Nonlinear Proc. Geophys., 10(6), 525-529, 2003.

Rodriguez-Iturbe, I., De Power, F. B., Sharifi, M. B., and Georgakakos, K. P.: Chaos in rainfall, Water Resour. Res., 25(7), 1667-1675, 1989.

Schertzer, D., Tchiguirinskaia, I., Lovejoy, S., Hubert, P., Bendjoudi, H., and Larcheveque, M.: Which chaos in the rainfallrunoff process? Discussion of "Evidence of chaos in the rainfallrunoff process?”, Hydrol. Sci. J., 47(1), 139-147, 2002.

Sivakumar, B.: Chaos theory in hydrology: important issues and interpretations. J. Hydrol., 227(1-4), 1-20, 2000.

Sivakumar, B.: Chaos theory in geophysics: Past, present and future, Chaos Soliton. Fract., 19(2), 441-462, 2004.

Sivakumar, B., Sorooshian, S., Gupta, H. V., and Gao, X.: A chaotic approach to rainfall disaggregation, Water Resour. Res., 37(1), 61-72, 2001

Sivakumar, B., Berndtsson, R., Olsson, J., Jinno, K.: Reply to "Which chaos in the rainfall-runoff process?", Hydrol. Sci. J., 47(1), 149-158, 2002a.

Sivakumar, B., Persson, M., Berndtsson, R., and Uvo, C. B.: Is correlation dimension a reliable indicator of low-dimensional chaos in short hydrological time series?, Water Resour. Res., 38(2), 1011, doi:10.1029/2001WR000333, 2002b.

Takens, F.: Detecting strange attractors in turbulence, in: Dynamical Systems and Turbulence, edited by: Rand, D. A. and Young, L. S., Lecture Notes in Mathematics 898, SpringerVerlag, Berlin, Germany, 366-381, 1981.

Tsonis, A. A., Elsner, J. B., and Georgakakos, K. P.: Estimating the dimension of weather and climate attractors: important issues about the procedure and interpretation, J. Atmos. Sci., 50, 25492555, 1993.

Veneziano, D., Moglen, G. E., Furcolo, P., and Iacobellis, V.: Stochastic model of the width function, Water Resour. Res. 36(4), 1143-1158, 2000.

Wainwright, J. and Mulligan, M. (Eds.): Environmental Modeling - Finding Simplicity in Complexity, John Wiley \& Sons, United Kingdom, 2004. 